\title{
Cauchy Problem for a Nonstrictly Hyperbolic Equation on a Half-Plane with Constant Coefficients
}

\author{
V. I. Korzyuk, I. S. Kozlovskaya, and A. I. Kozlov \\ Institute of Mathematics, National Academy of Sciences, Minsk, Belarus \\ Belarus State University, Minsk, Belarus \\ e-mail:korzyuk@bsu.by,kozlovskaja@bsu.by,fpm.kozlovai@bsu.by
}

Received June 2, 2014

\begin{abstract}
We consider the Cauchy problem for a nonstrictly hyperbolic equation of arbitrary order with constant coefficients. The operator in the equation is a composition of first-order differential operators. The equation is supplemented with Initial conditions. We find the solution of this problem on a half-plane in analytic form in the case of two independent variables under some conditions on the coefficients.
\end{abstract}

DOI: $10.1134 /$ S0012266115060038

\section{INTRODUCTION}

In the present paper, we consider the Cauchy problem on a half-plane for a nonstrictly hyperbolic linear equation of order $m$ with constant coefficients, where $m$ is a positive integer. The operator in the equation is a composition of first-order differential operators. The Cauchy problem for such an equation was earlier considered in $[1,2]$ in the case of a strictly hyperbolic equation (a Petrovskii hyperbolic equation $[3,4]$ ). The general solution of both strictly and nonstrictly hyperbolic equations of arbitrary order was constructed there as well. The case of a nonstrictly hyperbolic equation with the coincidence of all characteristics was considered in [5], and the solutions of the Cauchy problem in all cases of a nonstrictly hyperbolic third-order equation of such a form were obtained in $[6]$.

\section{STATEMENT OF THE PROBLEM}

On the plane $\mathbb{R}^{2}$ of two independent variables $t$ and $x$, we introduce the half-plane $Q=(0, \infty) \times \mathbb{R}$ on which we consider the following differential equation of order $m \in N$, where $N$ is the set of positive integers, for a function $u: \mathbb{R}^{2} \supset Q \ni(t, x) \rightarrow u(t, x) \in \mathbb{R}$ :

$$
\mathfrak{L}^{(m)} u=\prod_{k=1}^{m}\left(\partial_{t}-a^{(k)} \partial_{x}+b^{(k)}\right) u(t, x)=f(t, x), \quad(t, x) \in Q,
$$

where $\partial_{t}=\partial / \partial t$ and $\partial_{x}=\partial / \partial x$ are the first derivatives with respect to $t$ and $x, a^{(k)}$ and $b^{(k)}$ are given real numbers, $f: \mathbb{R}^{2} \supset \bar{Q} \ni(t, x) \rightarrow f(t, x) \in \mathbb{R}$ is a given function on $\bar{Q}$, and $\bar{Q}$ is the closure of the domain $Q$; i.e., $\bar{Q}=[0, \infty) \times \mathbb{R}$. The Cauchy conditions

$$
\partial_{t}^{j} u(0, x)=\varphi^{(j)}(x), \quad x \in \mathbb{R}, \quad j=0, \ldots, m-1,
$$

are posed on the boundary

$$
\partial Q=\{(t, x) \in \bar{Q} \mid t=0\}
$$

of the domain $Q$, where $\partial_{t}^{j}$ stands for the derivative $\partial^{j}=\partial^{j} / \partial t^{j}$. 


\section{PROBLEM IN THE CASE OF EQUAL COEFFICIENTS}

Consider the $m$ th-order homogeneous equation

$$
\mathfrak{L}^{(m)} u(t, x)=0, \quad(t, x) \in Q,
$$

for an operator of the general form

$$
\mathfrak{L}^{(m)}=\prod_{k=1}^{p}\left(\partial_{t}-a^{(k)} \partial_{x}+b^{(k)}\right)^{r(k)},
$$

where $p$ and $r(k)$ are positive integers such that $r(1)+\cdots+r(p)=m$. By [2], the general solution of Eq. (3.1) has the form

$$
u(t, x)=\sum_{k=1}^{p} e^{-b^{(k)}} t \sum_{s=1}^{r(k)} t^{s-1} f^{(k s)}\left(x+a^{(k)} t\right),
$$

where the $f^{(k s)}: \mathbb{R} \rightarrow \mathbb{R}$ are arbitrary functions differentiable up to order $m$.

Consider the case of the operator (3.2) in which $p=1, r(1)=m, a^{(k)}=a$, and $b^{(k)}=b$ for all $k=1, \ldots, m, a, b \in \mathbb{R}$.

By $C^{l}(G)$ we denote the set of functions continuous together with their continuously differentiable derivatives up to order $l$ defined on the set $G$. In what follows, for $G$ we take the sets $\bar{Q}$ and $\mathbb{R}$.

Theorem 3.1. Let

$$
\mathfrak{L}^{(m)} u=\left(\partial_{t}-a \partial_{x}+b\right)^{m} u=0, \quad a, b \in \mathbb{R} .
$$

Then the Cauchy problem (3.4), (2.2) has a unique classical solution in $C^{m}(\bar{Q})$ for arbitrary functions $\varphi^{(j)}$ in the classes $C^{2 m-j-1}(\mathbb{R}), j=0, \ldots, m-1$.

Proof. By formula (3.3), the general classical solution of Eq. (3.4) has the form

$$
u(t, x)=e^{-b t} \sum_{k=0}^{m-1} t^{k} f^{(k)}(x+a t),
$$

where the $f^{(k)}$ are arbitrary real functions in the class $C^{m}(\mathbb{R})$.

Let us compute the derivatives of the function (3.5) of order $j$ with respect to $t$ :

$$
\begin{aligned}
\partial_{t}^{j} u= & e^{-b t}\left(\sum_{k=0}^{j-1} \sum_{i=0}^{j} Q_{k i}(t) \partial_{x}^{i} f^{(k)}(x+a t)\right. \\
& \left.+\sum_{k=j}^{m-1} \sum_{i=0}^{j} P_{k i}(t) t^{k+i-j} \partial_{x}^{i} f^{(k)}(x+a t)\right), \quad j=1, \ldots, m-1,
\end{aligned}
$$

where $m \geq 2$, the $Q_{k i}(t)$ are polynomials of degree $\leq k$, the $P_{k i}(t)$ are polynomials of degree $\leq j-i$, and $P_{k 0}(0)=k ! /(k-j)$ !. If $j=0$, then we have the function (3.5). For other values of $j$, we prove the expression (3.6) by induction.

If $j=1$, then

$$
\begin{aligned}
\partial_{t} u(t, x)= & e^{-b t}\left(-b f^{(0)}(x+a t)+a \partial_{x} f^{(0)}(x+a t)\right. \\
& \left.+\sum_{k=1}^{m-1}\left(\left(-b t^{k}+k t^{k-1}\right) f^{(k)}(x+a t)+a t^{k} \partial_{x} f^{(k)}(x+a t)\right)\right),
\end{aligned}
$$

DIFFERENTIAL EQUATIONS Vol. $51 \quad$ No. $6 \quad 2015$ 
where, in our notation, the polynomials have the form $Q_{00}(t)=-b, Q_{01}(t)=a, P_{k 0}(t)=-b t+k$, and $P_{k 1}(t)=a$; in addition, $P_{k 0}(0)=k=k ! /(k-1)$ !, which corresponds to formula (3.6).

Now let the representation (3.6) be valid for some $j \geq 1, j \in N$. Then

$$
\begin{aligned}
\partial_{t}^{j+1} u(t, x)= & \partial_{t}\left(\partial_{t}^{j} u\right)(t, x) \\
= & \partial_{t}\left[e^{-b t}\left(\sum_{k=0}^{j-1} \sum_{i=0}^{j} Q_{k i}(t) \partial_{x}^{i} f^{(k)}(x+a t)+\sum_{k=j}^{m-1} \sum_{i=0}^{j} P_{k i}(t) t^{k+i-j} \partial_{x}^{i} f^{(k)}(x+a t)\right)\right] \\
= & e^{-b t}\left[\sum_{k=0}^{j} \sum_{i=0}^{j+1} \widetilde{Q}_{k i}(t) \partial_{x}^{i} f^{(k)}(x+a t)+\sum_{k=j+1}^{m-1} \sum_{i=0}^{j}\left(\left(-b t P_{k i}(t)+t P_{k i}^{\prime}(t)\right.\right.\right. \\
& \left.\left.\left.+(k+i-j) P_{k i}(t)\right) t^{k+i-j-1} \partial_{x}^{i} f^{(k)}(x+a t)+a P_{k i}(t) t^{k+(i+1)-(j+1)} \partial_{x}^{i+1} f^{(k)}(x+a t)\right)\right] \\
= & e^{-b t}\left[\sum_{k=0}^{j} \sum_{i=0}^{j+1} \widetilde{Q}_{k i}(t) \partial_{x}^{i} f^{(k)}(x+a t)+\sum_{k=j+1}^{m-1} \sum_{i=0}^{j+1} \widetilde{P}_{k i}(t) t^{k+i-j-1} \partial_{x}^{i} f^{(k)}(x+a t)\right],
\end{aligned}
$$

where $\widetilde{P}_{k 0}(0)=(k-j) P_{k 0}(0)=k ! /(k-(j+1)) !$. Here we have used the fact that the formula

$$
\partial_{t}\left(e^{-b t} \sum_{i=0}^{j} R_{k i}(t) \partial_{x}^{i} f(x+a t)\right)=e^{-b t} \sum_{i=0}^{j+1} \widetilde{R}_{k i}(t) \partial_{x}^{i} f(x+a t)
$$

holds for arbitrary polynomials $R_{k i}$ of degree $\leq k$ and for any function $f \in C^{j+1}(\mathbb{R})$.

Therefore, formula (3.6) holds for the $j$ th derivative, $j=1, \ldots, m-1$, of the function $u$ with respect to the variable $t$. By substituting the expressions (3.5) and (3.6) into the Cauchy conditions (2.2), we obtain a system of equations for the functions $f^{(k)}(x), k=0, \ldots, m-1$, of the form

$$
u(0, x)=f^{(0)}(x)=\varphi^{(0)}(x), \quad \partial_{t}^{j} u(0, x)=\sum_{k=0}^{j} \sum_{i=0}^{j-k} C^{(j k i)} \partial_{x}^{i} f^{(k)}(x)=\varphi^{(j)}(x), \quad j=1, \ldots, m-1 .
$$

By solving the resulting system successively, we find all values $f^{(k)}(x)$ of the function $f^{(k)}, k=$ $0, \ldots, m-1$. One can readily see that the functions $f^{(k)}$ are uniquely determined via the functions $\varphi^{(j)}$ and their derivatives, $k, j \in\{0,1, \ldots, m-1\}$. Then by formula (3.5) we find the unique classical solution $u$ of the Cauchy problem $(3.4),(2.2)$ in $C^{m}(\bar{Q})$ if the given functions $\varphi^{(j)}, j=0, \ldots, m-1$, belong to the corresponding classes $C^{2 m-j-1}(\mathbb{R})$. The proof of the theorem is complete.

In particular, by solving system (3.8) for $m=3$ and by substituting the obtained result into relation (3.5), we obtain the solution of the Cauchy problem (3.4), (2.2) in the form

$$
\begin{aligned}
u(t, x)= & e^{-b t}\left[\frac{t^{2}}{2}\left(\varphi^{(2)}-2 a \varphi^{(1)^{\prime}}+a^{2} \varphi^{(0)^{\prime \prime}}+2 b \varphi^{(1)}-2 a b \varphi^{(0)^{\prime}}-b^{2} \varphi^{(0)}\right)(x+a t)\right. \\
& \left.+t\left(\varphi^{(1)}-a \varphi^{(0)^{\prime}}+b \varphi^{(0)}\right)(x+a t)+\varphi^{(0)}(x+a t)\right] .
\end{aligned}
$$

Now consider the Cauchy problem for the inhomogeneous equation in the case of equal coefficients; i.e., let us find the solution of the equation

$$
\mathfrak{L}^{(m)} u=\left(\partial_{t}-a \partial_{x}+b\right)^{m} u(t, x)=f(t, x), \quad(t, x) \in Q,
$$

with the Cauchy conditions (2.2). 
Since the considered problem is linear, it follows that its solution $u$ can be represented as the sum of two functions $u=\tilde{u}+v$, where $\tilde{u}$ is a solution of problem (3.4), (2.2), and $v$ is a solution of Eq. (3.9) with the homogeneous Cauchy conditions

$$
\partial_{t}^{j} v(0, x)=0, \quad j=0, \ldots, m-1 .
$$

We define the function $v$ via the function $w: \mathbb{R}^{3} \supset[0, \infty) \times[0, \infty) \times \mathbb{R} \ni(t, \tau, x) \rightarrow w(t, \tau, x) \in \mathbb{R}$ with a parameter $\tau \in[0, \infty)$ by the relation

$$
v(t, x)=\int_{0}^{t} w(t-\tau, \tau, x) d \tau
$$

The function $w$ treated as a function of the independent variables $t$ and $x$ is a solution of the homogeneous equation (3.4) with the Cauchy conditions

$$
\partial_{t}^{j} w(0, \tau, x)=0, \quad j=0, \ldots, m-2, \quad \partial_{t}^{m-1} w(0, \tau, x)=f(\tau, x),
$$

where $f$ is the right-hand side of Eq. (3.9). To this end, we assume that the function $f$ belongs to the set $C^{0, m}(\bar{Q})$, where $C^{0, m}(\bar{Q})$ is the set of continuous functions defined on $\bar{Q}$ and continuously differentiable with respect to the variable $x$.

By virtue of condition (3.12), the function $v$ defined by (3.11) satisfies the Cauchy condition (3.10). Indeed, we have

$$
\partial_{t}^{j} v(t, x)=\partial_{t}^{j-k} \int_{0}^{t} \partial_{t}^{k} w(t-\tau, \tau, x) d \tau, \quad k=0, \ldots, j, \quad j=0, \ldots, m-1 .
$$

This, together with conditions (3.12), implies the relations

$$
\partial_{t}^{j} v(t, x)=\int_{0}^{t} \partial_{t}^{j} w(t-\tau, \tau, x) d \tau
$$

By setting $t=0$ in relation (3.13), we obtain condition (3.10) for the function $v$.

Let us show that the function $v$ defined in (3.11) satisfies Eq. (3.9); in this connection, we use the fact that $w$ is a solution of problem (3.4), (3.12) and

$$
\mathfrak{L}^{(m)}=\partial_{t}^{m}+m\left(-a \partial_{x}+b\right) \partial_{t}^{m-1}+\cdots+\left(-a \partial_{x}+b\right)^{m} .
$$

Therefore,

$$
\mathfrak{L}^{(m)} v(t, x)=\mathfrak{L}^{(m)} \int_{0}^{t} w(t-\tau, \tau, x) d x=\partial_{t} \int_{0}^{t} f(\tau, x) d \tau+\int_{0}^{t} \mathfrak{L}^{(m)} w(t-\tau, \tau, x) d x=f(t, x) ;
$$

in addition,

$$
w(t, \tau, x)=e^{-b t} t^{m-1} f(\tau, x+a t)
$$

and

$$
v(t, x)=\int_{0}^{t} e^{-b(t-\tau)}(t-\tau)^{m-1} f(\tau, x+a(t-\tau)) d \tau .
$$

The last results, together with Theorem 3.1, imply the following assertion.

DIFFERENTIAL EQUATIONS $\quad$ Vol. $51 \quad$ No. $6 \quad 2015$ 
Theorem 3.2. If the right-hand side of Eq. (3.9) belongs to the set $C^{0, m}(\bar{Q})$ and the functions $\varphi^{(j)}$ occurring in condition $(2.2)$ belong to the class $C^{2 m-j-1}(\mathbb{R})$, then for arbitrary such functions, there exists a unique classical solution $u=\tilde{u}+v$ of problem $(3.9),(2.2)$ in the class $C^{m}(\bar{Q})$, where $\tilde{u}$ is the classical solution of problem (3.4), (2.2) and $v$ is the solution of problem (3.9), (3.10).

\section{THE CASE OF PAIRWISE DISTINCT COEFFICIENTS}

In this section, we consider the nonstrictly hyperbolic equation

$$
\mathfrak{L}^{(m)} u(t, x)=f(t, x)
$$

of order $m$, where the coefficients of the operator $\mathfrak{L}^{(m)}$ are pairwise distinct. It follows that $\left(a^{(k)}, b^{(k)}\right) \neq\left(a^{(j)}, b^{(j)}\right)$ for all $k, j \in\{1, \ldots, m\}$ and $k \neq j$, or

$$
\left(a^{(k)}-a^{(j)}\right)^{2}+\left(b^{(k)}-b^{(j)}\right)^{2} \neq 0
$$

for $k \neq j$. Let the operator $\mathfrak{L}^{(m)}$ have the form

$$
\mathfrak{L}^{(m)}=\prod_{k=1}^{m}\left(\partial_{t}-a^{(k)} \partial_{x}+b^{(k)}\right) .
$$

For this operator, consider the homogeneous equation

$$
\mathfrak{L}^{(m)} u(t, x)=\prod_{k=1}^{m}\left(\partial_{t}-a^{(k)} \partial_{x}+b^{(k)}\right) u(t, x)=0 .
$$

By (3.3), the general solution of Eq. (4.3) has the form

$$
u(t, x)=\sum_{k=1}^{m} e^{-b^{(k)} t} f^{(k)}\left(x+a^{(k)} t\right)
$$

where the $f^{(k)}$ are arbitrary functions in the class $C^{m}(\mathbb{R})$.

Let us indicate the construction of the solution of problem (4.3), (2.2). To this end, in the representation (4.4), one should find the functions $f^{(k)}, k=1, \ldots, m$, via the given functions $\varphi^{(k)}$ $(k=0, \ldots, m-1)$ occurring in the initial conditions $(2.2)$.

We introduce the following notation of differential operators: $d_{k}=a_{k} d / d x-b^{(k)}$ and $d_{k}^{j}=$ $\left(a_{k} d / d x-b^{(k)}\right)^{j}$. In this notation, we rewrite the Cauchy conditions (2.2) for the unknown function (4.4) in the form of the system of differential equations

$$
\begin{aligned}
f^{(1)}(x)+f^{(2)}(x)+\cdots+f^{(m)}(x) & =\varphi^{(0)}(x), \\
d_{1} f^{(1)}(x)+d_{2} f^{(2)}(x)+\cdots+d_{m} f^{(m)}(x) & =\varphi^{(1)}(x), \quad \ldots, \\
d_{1}^{m-1} f^{(1)}(x)+d_{2}^{m-1} f^{(2)}(x)+\cdots+d_{m}^{m-1} f^{(m)}(x) & =\varphi^{(m-1)}(x) .
\end{aligned}
$$

System (4.5) can be reduced to a system of differential equations with diagonal matrix. To this end, we apply the operator $d_{m}$ to the first $m-1$ equations in system (4.5) and subtract every other equation of the resulting system from the preceding one. As a result, we obtain a new system of the form

$$
\begin{aligned}
f^{(1)}(x)+\cdots+f^{(m)}(x) & =\varphi^{(0)}(x), \\
\left(d_{1}-d_{m}\right) f^{(1)}(x)+\cdots+\left(d_{m-1}-d_{m}\right) f^{(m-1)}(x) & =\varphi^{(1)}(x)-d_{m} \varphi^{(0)}(x), \\
\left(d_{1}^{2}-d_{1} d_{m}\right) f^{(1)}(x)+\cdots+\left(d_{m-1}^{2}-d_{m-1} d_{m}\right) f^{(m-1)}(x) & =\varphi^{(2)}(x)-d_{m} \varphi^{(1)}(x), \quad \ldots, \\
\left(d_{1}^{m-1}-d_{1}^{m-2} d_{m}\right) f^{(1)}(x)+\cdots+\left(d_{m-1}^{m-1}-d_{m-1}^{m-2} d_{m}\right) f^{(m-1)}(x) & =\varphi^{(m-1)}(x)-d_{m} \varphi^{(m-2)}(x) .
\end{aligned}
$$


Next, we apply the operator $d_{m-1}$ to both sides in each equation starting from the second one in system (4.6) and perform similar subtractions from each other.

By continuing transformations in a similar way, we obtain the system of differential equations

$$
\begin{aligned}
& f^{(1)}(x)+\cdots+f^{(m)}(x)=\varphi^{(0)}(x)=\Phi^{(0)}(x), \\
& \left(d_{1}-d_{m}\right) f^{(1)}(x)+\cdots+\left(d_{m-1}-d_{m}\right) f^{(m-1)}(x)=\varphi^{(1)}(x)-d_{m} \varphi^{(0)}(x)=\Phi^{(1)}(x), \\
& \left(d_{1}-d_{m}\right)\left(d_{1}-d_{m-1} f^{(1)}(x)+\cdots+\left(d_{m-2}-d_{m}\right)\left(d_{m-2}-d_{m-1}\right) f^{(m-2)}(x)\right. \\
& =\varphi^{(2)}(x)-d_{m-1} \varphi^{(1)}(x)-d_{m-1} d_{m} \varphi^{(0)}(x)=\Phi^{(2)}(x), \quad \cdots, \\
& \left(d_{1}-d_{m}\right) \cdots\left(d_{1}-d_{2}\right) f^{(1)}(x)=\Phi^{(m-1)}(x) .
\end{aligned}
$$

If we assume that $\varphi^{(j)} \in C^{m-1-j}(\mathbb{R})$, then the $\Phi^{(j)}, j=0, \ldots, m-1$, are continuous functions. Next, since $d_{k} \neq d_{j}$ for $k \neq j$, it follows that, by solving system (4.7) upwards, we find solutions $f^{(k)}(k=1, \ldots, m)$ that are jointly determined to within $m(m-1) / 2$ constants in the general case. By defining the functions $f^{(k)}$ by formula (4.4), we define a solution of the Cauchy problem (4.3), (2.2).

Now consider the set of coefficient pairs $S=\left(a^{(i)}, b^{(i)}\right)$, $\operatorname{card}(S)=m$. Two pairs of coefficients $\left(a^{(i)}, b^{(i)}\right)$ and $\left(a^{(j)}, b^{(j)}\right)$ are said to be equivalent if $a^{(i)}=a^{(j)}$. The set $S$ thereby splits into equivalence classes $S_{k}=\left(a_{k}, b_{k}\right)$. Let card $\left(S_{k}\right)=A^{(k)}$, and let the number of such equivalence classes be equal to $\operatorname{card}(S / \sim)=q$. Then $\sum_{i=1}^{q} A^{(i)}=m$.

Let us show that the smoothness condition $\varphi^{(j)} \in C^{2 m-q-j}(\mathbb{R})$ for the function $\varphi^{(j)}$ is sufficient for the solvability of system (4.7). In this connection, we need the following assertion.

Lemma 4.1. Let the function $\Phi$ belong to $C^{k}(\mathbb{R})$, let $\alpha^{(i)} \in \mathbb{R}, i=0, \ldots, m$, be constant coefficients, and let $\alpha^{(m)} \neq 0$. Then there exists a solution of the ordinary differential equation

$$
D^{(m)} f=\left(\alpha^{(m)} \partial_{x}^{m}+\alpha^{(m-1)} \partial_{x}^{m-1}+\cdots+\alpha^{(0)}\right) f=\Phi(x) ;
$$

moreover, $f \in C^{m+k}(\mathbb{R})$.

Proof. The existence of a solution of the above-represented equation is a well-known fact in the theory of ordinary differential equations. Now let us show that any solution $f$ of this equation is a $m+k$ times continuously differentiable function. Let $f \in C^{n}(\mathbb{R})$, but, in addition, let $f \notin C^{n+1}(\mathbb{R})$. It is known that $m \leq n$. Suppose that $n<m+k$. Then $\partial_{x}^{j} f \in C^{n-j}(\mathbb{R})$ but $\partial_{x}^{j} f \notin C^{n-j+1}(\mathbb{R})$. We consider the following identity, which is obtained by the substitution of the solution $f$ of this equation:

$$
\alpha^{(m)} \partial_{x}^{m} f=\Phi-\alpha^{(m-1)} \partial_{x}^{m-1} f-\cdots-\alpha^{(0)} f .
$$

One can readily see that the right-hand side in the identity belongs to $C^{n-m+1}(\mathbb{R})$, while $\alpha^{(m)} \partial_{x}^{m} f \notin$ $C^{n-m+1}(\mathbb{R})$. It follows from the resulting contradiction that $n \geq m+k$; therefore, $f \in C^{m+k}(\mathbb{R})$. The proof of the lemma is complete.

Now consider system (4.5). Without loss of generality, we arrange the functions $f^{(i)}$ (respectively, the coefficients $a^{(i)}$ and $b^{(i)}$ of the operator $L^{(m)}$ ) of this system so as to ensure that functions corresponding to coefficients in one equivalence class follow each other. In other words, if $\left(a^{(i)}, b^{(i)}\right) \in S_{j}$, then $\left(a^{(i+1)}, b^{(i+1)}\right) \in S_{j}$ or $\left(a^{(i+1)}, b^{(i+1)}\right) \in S_{j+1}$.

Now consider the matrix Ord $\in \operatorname{Matr}_{m \times m}(\mathbb{Z})$ in which the order of the differential operator in the intersection of the $i$ th row and the $j$ th column is equal to the order of the operator multiplying $f^{(j)}$ in the $i$ th equation in (4.5). We also consider the column $\Psi \in \mathbb{Z}^{m}$ whose $i$ th element stands for the smoothness order of the right-hand side in the $i$ th equation. Then originally Ord and $\Psi$ have the form

$$
\operatorname{Ord}=\left(\begin{array}{ccccc}
0 & 0 & \ldots & 0 & 0 \\
1 & 1 & \ldots & 1 & 1 \\
\ldots & \ldots & \ldots & \ldots & \ldots \\
m-1 & m-1 & \ldots & m-1 & m-1
\end{array}\right), \quad \Psi=\left(\begin{array}{c}
2 m-q \\
2 m-q-1 \\
\ldots \\
m-q+1
\end{array}\right)
$$

DIFFERENTIAL EQUATIONS Vol. $51 \quad$ No. $6 \quad 2015$ 
Under the passage from system (4.5) to system (4.7), the order of differential operators in each of equations does not grow and only can diminish for the case in which $d_{j}$ is subtracted from $d_{i}$ and $a^{(i)}=a^{(j)}$. Likewise, the smoothness of the right-hand side of the system does not diminish under the passage to an upper triangular form. After transformations, the matrix Ord and the column $\Psi$ acquire the form

$$
\operatorname{Ord}=\left(\operatorname{Ord}_{1}, \operatorname{Ord}_{2}, \ldots, \operatorname{Ord}_{q}\right), \quad \Psi=\left(\begin{array}{c}
2 m-q \\
2 m-q-1 \\
\ldots \\
m-q+1
\end{array}\right)
$$

where the $\operatorname{Ord}_{i}$ are blocks of dimensions $A_{i} \times m$ corresponding to equivalence classes of coefficients and have the form

$$
\operatorname{Ord}_{q}=\left(\begin{array}{cccc}
0 & \ldots & 0 & 0 \\
0 & \ldots & 0 & - \\
\ldots & \ldots & \ldots & \ldots \\
0 & \ldots & - & - \\
- & \ldots & - & - \\
\ldots & \ldots & \ldots & \ldots \\
- & \ldots & - & -
\end{array}\right), \quad \operatorname{Ord}_{i}=\left(\begin{array}{cccc}
0 & \ldots & 0 & 0 \\
1 & \ldots & 1 & 1 \\
\ldots & \ldots & \ldots & \ldots \\
\sum_{j=i+1}^{q} A^{(j)} & \ldots & \sum_{j=i+1}^{q} A^{(j)} & \sum_{j=i+1}^{q} A^{(j)} \\
\sum_{j=i+1}^{q} A^{(j)} & \ldots & \sum_{j=i+1}^{q} A^{(j)} & - \\
\ldots & \ldots & \ldots & \ldots \\
\sum_{j=i+1}^{q} A^{(j)} & \ldots & - & - \\
\ldots & \ldots & \ldots & \ldots \\
- & \ldots & - & -
\end{array}\right),
$$

The symbol "_" implies that the corresponding function is eliminated from the equation. In each row of each block, the order of differential operators is increased by 1 until the corresponding functions are eliminated. When functions are eliminated for one equivalence class, the order of differential operators is preserved. This can readily be traced, because $d_{i}-d_{j}=b_{j}-b_{i} \in \mathbb{R}$ provided that $f^{(i)}$ and $f^{(j)}$ correspond to one equivalence class.

By solving system (4.7) upwards and by taking into account Lemma 4.1, we obtain functions $f^{(j)}$ with the following smoothness degrees:

$$
\begin{aligned}
& f^{(j)} \in C^{2 m-q-\left(A^{(1)}-1\right)}(\mathbb{R}), \quad j=1, \ldots, A^{(1)}, \\
& f^{(j)} \in C^{2 m-q-\left(A^{(1)}-1\right)-\left(A^{(2)}-1\right)}(\mathbb{R}), \quad j=A^{(1)}+1, \ldots, A^{(1)}+A^{(2)}, \quad \ldots, \\
& f^{(j)} \in C^{2 m-q-\sum_{i=1}^{q}\left(A^{(i)}-1\right)}(\mathbb{R})=C^{m}(\mathbb{R}), \quad j=m-A^{(q)}+1, \ldots, m .
\end{aligned}
$$

Therefore, $f^{(j)} \in C^{m}(\mathbb{R}), j=1, \ldots, m$, and, after the substitution of $f^{(j)}$ into the general solution formula, we find that the general solution $u(t, x)$ belongs to $C^{m}(\bar{Q})$. Consequently, the conditions imposed on the smoothness of the functions $\varphi^{(j)}, j=0, \ldots, m-1$, are sufficient.

We have thereby proved the following assertion.

Theorem 4.1. If $\varphi^{(j)} \in C^{2 m-q-j}(\mathbb{R}), j=0, \ldots, m-1$, and condition (4.2) holds for the coefficients of Eq. (4.3), then there exists a classical solution $u \in C^{m}(\bar{Q})$ of the form (4.4) for problem (4.3), (2.2), where the functions $f^{(k)}(k=1, \ldots, m)$ are solutions of the system of differential equations (4.7). 
As was mentioned above, the solutions $f^{(k)}(k=1, \ldots, m)$ of system $(4.7)$ are solutions of differential equations and hence are determined to within arbitrary constants. But, by formula (4.4), in total they define the unique solution. The last assertion should be proved. We simultaneously consider the case of the inhomogeneous equation (4.1) as well.

Theorem 4.2. If $\varphi^{(j)} \in C^{2 m-q-j}(\mathbb{R}), j=0, \ldots, m-1, f \in C^{(0, m-q+1)}(\bar{Q})$, and the coefficients of Eq. (4.1) satisfy condition (4.2), then there exists a unique classical solution $u \in C^{m}(\bar{Q})$ of problem (4.1), (4.2).

Proof. To prove the uniqueness of a classical solution of the Cauchy problem (4.1), (2.2), it suffices to show that there exists a unique solution $u(t, x) \equiv 0$ of the Cauchy problem for the homogeneous equation (4.3) with the homogeneous initial conditions

$$
\partial_{t}^{j} u(0, x) \equiv 0, \quad x \in \mathbb{R}, \quad j=0, \ldots, m-1 .
$$

The proof is by induction.

Let $b^{(i)} \neq b^{(j)}$ for all $i \neq j, i, j \in\{1, \ldots, m\}$, and $a^{(k)}=a$ for all $k=1, \ldots, m$. In this case, the uniqueness follows from Theorem 1 in [5] and from system (4.7), which can be represented as the algebraic system

$$
\begin{aligned}
f^{(1)}(x)+\cdots+f^{(m)}(x) & =0, \\
\left(b^{(1)}-b^{(2)}\right) f^{(1)}(x)+\cdots+\left(b^{(m-1)}-b^{(m)}\right) f^{(k)}(x) & =0, \quad \ldots, \\
\left(b^{(1)}-b^{(2)}\right) \cdots\left(b^{(1)}-b^{(m)}\right) f^{(1)}(x) & =0 .
\end{aligned}
$$

It follows from relations (4.9) that all functions $f^{(k)}(k=1, \ldots, m)$ defining the solution (4.4) of problem (4.3), (4.8) are zero. This implies the uniqueness of the solution of this problem for the case in which the coefficients $a^{(k)}(k=1, \ldots, m)$ coincide and the $b^{(k)}$ are distinct.

Therefore, for $q=1$ (the number of equivalence classes is equal to one), we have the uniqueness of the zero solution of problem (4.3), (4.8). Consider Eq. (4.3) of some order $m$ for which the set of coefficient pairs splits into more than one equivalence class; i.e., $q>1$.

Now, without loss of generality, we assume that $a^{(1)}=\cdots=a^{(s)}=a$ and $a^{(j)} \neq a$ for $j=$ $s+1, \ldots, m$, where $1 \leq s<m$. In addition, let $u(t, x)$ be some solution of problem (4.3), (4.8), whose existence is justified by Theorem 4.1.

Consider the system of identities that consists of two parts and is obtained as follows. The first part consists of the first $m-s$ equations in system (4.5),

$$
\begin{aligned}
f^{(1)}(x)+\cdots+f^{(s)}(x)+f^{(s+1)}(x)+\cdots+f^{(m)}(x) & \equiv 0, \ldots, \\
d_{1}^{m-s-1} f^{(1)}(x)+\cdots+d_{s}^{m-s-1} f^{(s)}(x)+d_{s+1}^{m-s-1} f^{(s+1)}(x)+\cdots+d_{m}^{m-s-1} f^{(m)}(x) & \equiv 0 .
\end{aligned}
$$

The second part of identities is obtained from the last $s$ equations of system (4.5) after a partial reduction to a system of the form (4.7); namely, after the elimination of the function $f^{(s+1)}$ from the last $s$ equations and after the reduction of the remaining equations, we obtain the system

$$
g^{(1)}(x)+\cdots+g^{(s)}(x) \equiv 0, \quad \cdots, \quad d_{1}^{s-1} g^{(1)}(x)+\cdots+d_{s}^{s-1} g^{(s)}(x) \equiv 0,
$$

where $g^{(j)}=\left(d_{j}-d_{s+1}\right) \cdots\left(d_{j}-d_{m}\right) f^{(j)}, j=1, \ldots, s$. Since, by assumption, $a^{(j)}=a, j=1, \ldots, s$, it follows from the assumptions of the theorem that $b^{(i)} \neq b^{(j)}$ for indices $i \neq j, i, j \in\{1, \ldots, s\}$. This, together with system (4.11), implies that

$$
g^{(j)}(x)=\left(d_{j}-d_{s+1}\right) \cdots\left(d_{j}-d_{m}\right) f^{(j)}(x) \equiv 0, \quad j=1, \ldots, s .
$$

By formula (4.4), in this case, the solution of problem (4.3), (4.8) can be represented as the sum of two functions $u=u^{(1)}+u^{(2)}$, where

$$
u^{(1)}(t, x)=\sum_{k=1}^{s} e^{-b^{(k)} t} f^{(k)}(x+a t), \quad u^{(2)}(t, x)=\sum_{k=s+1}^{m} e^{-b^{(k)} t} f^{(k)}\left(x+a^{(k)} t\right) .
$$


Let $\widetilde{u}^{(2)}(t, x)=-u^{(2)}(t, x)$. By virtue of identities (4.10), the function $\widetilde{u}^{(2)}$ is a solution of the problem

$$
\begin{aligned}
& \prod_{k=s+1}^{m}\left(\partial_{t}-a^{(k)} \partial_{x}+b^{(k)}\right) \tilde{u}^{(2)}(t, x)=0 \\
& \begin{aligned}
\partial_{t}^{j} \tilde{u}^{(2)}(0, x) & =-d_{1}^{j} f^{(s+1)}(x)-\cdots-d_{s}^{j} f^{(m)}(x)=d_{1}^{j} f^{(1)}(x)+\cdots+d_{s}^{j} f^{(s)}(x), \\
& \quad=0, \ldots, m-s-1 .
\end{aligned}
\end{aligned}
$$

Let us show that the function $u^{(1)}$ is also a solution of problem (4.14). Indeed, the Cauchy conditions of this problem hold for $u^{(1)}$ by virtue of the definition of the function $u^{(1)}$ by formula (4.13) via the functions $f^{(j)}, j=1, \ldots, s$.

One can readily see that

$$
\left(\partial_{t}-a^{(k)} \partial_{x}+b^{(k)}\right)\left(e^{-b^{(j)} t} f^{(j)}(x+a t)\right)=e^{-b^{(j)}} t\left(\left(d_{j}-d_{k}\right) f^{(j)}\right)(x+a t) .
$$

Therefore, by virtue of relations (4.12), we have

$$
\begin{aligned}
\prod_{k=s+1}^{m}\left(\partial_{t}\right. & \left.-a^{(k)} \partial_{x}+b^{(k)}\right)\left(e^{-b^{(j)} t} f^{(j)}(x+a t)\right) \\
& =e^{-b^{(j)} t}\left(\left(d_{j}-d_{s+1}\right) \cdots\left(d_{j}-d_{m}\right) f^{(j)}\right)(x+a t)=0, \quad j=1, \ldots, s .
\end{aligned}
$$

Hence we derive the relation

$$
\prod_{k=s+1}^{m}\left(\partial_{t}-a^{(k)} \partial_{x}+b^{(k)}\right) u^{(1)}(t, x) \equiv 0 .
$$

Therefore, the functions $u^{(1)}$ and $\tilde{u}^{(2)}=-u^{(2)}$ are solutions of the same Cauchy problem, which, by the induction assumption, has a unique solution $u^{(1)}=\tilde{u}^{(2)}$. Consequently, the solution of problem (4.3), (4.8) is $u=u^{(1)}+u^{(2)} \equiv 0$.

The assertion of Theorem 4.2 for problem (4.1), (2.2) in the case of an inhomogeneous equation can be proved by the scheme suggested in the proof of the final part of Theorem 3.2. The proof of the theorem is complete.

\section{GENERAL CASE OF EQ. (2.1), (3.2)}

Let us proceed to the study of the general case of a hyperbolic equation whose operator is a composition of first-order operators of the form (3.2). Let us first consider the Cauchy problem (3.1), (3.2), (2.2). Unlike previous sections, we only prove the existence and uniqueness of the classical solution. The general solution of Eq. (3.1), (3.2) can be represented by formula (3.3). Recall that $r(1)+\cdots+r(p)=m$ in (3.3) and $\left(a^{(i)}, b^{(i)}\right) \neq\left(a^{(j)}, b^{(j)}\right)$ only for $i \neq j$ in distinct blocks, i.e., for distinct indices $k$ in formula (3.3).

After the substitution of the expression (3.3) into the initial conditions (2.2), we obtain a system of equations that, for brevity, can be represented in the matrix form

$$
A f=\varphi
$$

where

$$
\begin{aligned}
& f=\left(f^{(11)}, \ldots, f^{(1 r(1))}, \ldots f^{(p 1)}, \ldots, f^{(p r(p))}\right)^{\mathrm{T}}, \quad \varphi=\left(\varphi^{(0)}, \ldots, \varphi^{(m-1)}\right)^{\mathrm{T}}, \\
& A=\operatorname{Matr}_{m \times m}, \quad A=\left(A_{1}, \ldots, A_{p}\right), \quad A_{j} \in \operatorname{Matr}_{m \times r(j)},
\end{aligned}
$$


CAUCHY PROBLEM FOR A NONSTRICTLY HYPERBOLIC EQUATION

$$
A_{j}=\left[\begin{array}{ccccc}
1 & 0 & 0 & \cdots & 0 \\
d_{j} & 1 ! & 0 & \cdots & 0 \\
d_{j}^{2} & \frac{2 !}{1 !} d_{j} & 1 ! & \cdots & 0 \\
\cdots & \cdots & \cdots & \cdots & \cdots \\
d_{j}^{r(j)-1} & \frac{(r(j)-1) !}{(r(j)-2) !} d_{j}^{r(j)-2} & \frac{(r(j)-1) !}{(r(j)-3) !} d_{j}^{r(j)-3} & \cdots & (r(j)-1) ! \\
\cdots & \cdots & \cdots & \cdots & \cdots \\
d_{j}^{m-1} & \frac{(m-1) !}{(m-2) !} d_{j}^{m-2} & \frac{(m-1) !}{(m-3) !} d_{j}^{m-3} & \cdots & \frac{(m-1) !}{(m-r(j)) !} d_{j}^{m-r(j)}
\end{array}\right],
$$

$j=1, \ldots, p, d_{j}=a^{(j)} \frac{d}{d x}-b^{(j)}$. Let us show that system (5.1) can be reduced to a system with an upper triangular matrix. The entries of the matrix are differential operators; they are treated as polynomials in the differentiation operator $d / d x$.

Lemma 5.1. Let $A=\left(A_{1}, \ldots, A_{p}\right)$ be a matrix over the ring of polynomials $\mathbb{R}[x]$, where

$$
A_{j}=\left[\begin{array}{ccccc}
1 & 0 & 0 & \cdots & 0 \\
Q_{j} & 1 ! & 0 & \cdots & 0 \\
Q_{j}^{2} & \frac{2 !}{1 !} Q_{j} & 1 ! & \ldots & 0 \\
\cdots & \cdots & \cdots & \cdots & \cdots \\
Q_{j}^{r(j)-1} & \frac{(r(j)-1) !}{(r(j)-2) !} Q_{j}^{r(j)-2} & \frac{(r(j)-1) !}{(r(j)-3) !} Q_{j}^{r(j)-3} & \ldots & (r(j)-1) ! \\
\cdots & \ldots & \cdots & \cdots & \ldots \\
Q_{j}^{m-1} & \frac{(m-1) !}{(m-2) !} Q_{j}^{m-2} & \frac{(m-1) !}{(m-3) !} Q_{j}^{m-3} & \cdots & \frac{(m-1) !}{(m-r(j)) !} Q_{j}^{m-r(j)}
\end{array}\right],
$$

$Q_{j} \in \mathbb{R}[z], Q_{j}=a^{(j)} x-b^{(j)}, a^{(j)}, b^{(j)} \in \mathbb{R},\left(a^{(i)}, b^{(i)}\right) \neq\left(a^{(j)}, b^{(j)}\right)$ for $i \neq j$. Then the determinant of the matrix $A$ is nonzero. In addition, the matrix $A$ can be reduced to upper-triangular form such that each $j$ th row contains polynomials of degree $\leq j-1$.

Proof. Consider the system of functions of the variable $t$, where $z$ is the expression

$$
\left\{t^{k} e^{Q_{j} t}, \quad k=0, \ldots, r_{j}-1, \quad j=1, \ldots, p\right\} .
$$

This function system is linearly independent. This implies that the Wronski determinant $W(t)$ of this system is nonzero at any point $t$, in particular, for $t=0$. Let us compute $W(0)$. We find the $j$ th derivative of the above-mentioned function system $t^{k} e^{Q_{i} t}$ :

$$
\frac{d^{j}}{d t^{j}}\left(t^{k} e^{Q_{i} t}\right)=\sum_{l=0}^{j}\left(\begin{array}{l}
j \\
l
\end{array}\right) \frac{d^{l}}{d t^{l}} t^{k} e^{Q_{i} t} Q_{i}^{j-l}
$$

where $\left(\begin{array}{l}j \\ l\end{array}\right)$ is the binomial coefficient. In the special case with $t=0$, it is given by the relation

$$
\left.\frac{d^{j}}{d t^{j}}\left(t^{k} e^{Q_{i} t}\right)\right|_{t=0}=\left\{\begin{array}{ll}
\left(\begin{array}{c}
j \\
k
\end{array}\right) k ! Q_{i}^{j-k} & \text { if } j \geq k, \\
0 & \text { if } j<k,
\end{array}= \begin{cases}\frac{j !}{(j-k) !} Q_{i}^{j-k} & \text { if } j \geq k, \\
0 & \text { if } j<k .\end{cases}\right.
$$

It follows from the last relations that the Wronski determinant of the function system (5.2) is the determinant of the matrix $A$, i.e., $\operatorname{det} A=W(0)$. 
We have thereby shown that $\operatorname{det} A \neq 0$. Since $A$ is a nonsingular matrix, we find that it can be reduced to an upper-triangular form by means of elementary transformations, i.e., the multiplication of a row by a nonzero scalar and the subtraction of one row multiplied by a polynomial from another. These transformations do not increase the maximum degree of polynomials in a matrix row.

It remains to note that the $j$ th row of the matrix $A$ contains originally polynomials of degree $\leq j-1$. This implies that this invariant is preserved under the reduction of the matrix $A$ to upper-triangular form. The proof of the lemma is complete.

It directly follows from Lemma 5.1 that the system of differential equations (5.1) can be reduced to upper-triangular form; in this case, the order of the $j$ th equation does not exceed $j-1$, and the differential operator on the diagonal is nonzero. By solving the obtained system of ordinary differential equations upwards, we define a solution of the Cauchy problem (3.1), (3.2), (2.2). If Eq. (2.1) is inhomogeneous, i.e., if we deal with the Cauchy problem (2.1), (3.2), (2.2), then we obtain its solution in the form of the sum of a solution of problem (3.1), (3.2), (2.2) and a particular solution $v$ defined by formula (3.11), where $w$ is a solution of Eq. (3.1), (3.2) satisfying condition (3.12).

Theorem 5.1. Let the following condition be satisfied:

$$
f \in C^{(0, m)}(\bar{Q}), \quad \varphi^{(j)} \in C^{2 m-j-1}(\mathbb{R}), \quad j=0, \ldots, m-1, \quad a^{(k)}, b^{(k)} \in \mathbb{R} .
$$

Then there exists a solution $u: \mathbb{R}^{2} \supset \bar{Q} \ni(t, x) \rightarrow u(t, x) \in \mathbb{R}$ of the Cauchy problem (2.1), (3.2), (2.2).

Proof. The desired assertion follows from Lemma 5.1 and the preceding argument.

Note also that the functions $f^{(1 s)}$ depend at most on $s-1, s=1, \ldots, r(1)$, constants. Since the blocks $A_{k}$ of the matrix $A$ of system (5.1) can be arranged in any order, it follows from the symmetry that the functions $f^{(k s)}$ depend at most on $s-1$ constants, where $s=1, \ldots, r(k)$ and $k=1, \ldots, p$. Then, in the whole, the solution $u(t, x)$ of problem $(3.1),(3.2),(2.2)$ depends on at most

$$
\sum_{k=1}^{p} \frac{r(k)(r(k)-1)}{2} \leq \frac{m(m-1)}{2}
$$

constants.

Let us prove the uniqueness of the classical solution of problem (2.1), (3.2), (2.2). Since the considered problem is linear, we find that the uniqueness of its solution is a consequence of the fact that the corresponding homogeneous problem (3.1), (3.2), (4.8) has only the zero solution $u(t, x) \equiv 0$.

The latter assertion be proved by induction. Let $m=1$. In this case, Eq. (3.1) is the linear first-order differential equation

$$
\left(\partial_{t}-a \partial_{x}+b\right) u=0 .
$$

The general solution of Eq. (5.3) has the form

$$
u(t, x)=e^{-b t} f^{(m)}(x+a t) .
$$

If the Cauchy condition is homogeneous, $u(0, x)=0$, then from formula (5.4), we obtain the solution $u(t, x) \equiv 0$.

Suppose that, for any $m<n, m, n \in N$, problem (3.1), (3.2), (4.8) has only the trivial solution. Let us show that this problem has only the trivial solution $u(t, x) \equiv 0$ for $m=n$ as well.

By Theorem 5.1, there exists a classical solution $u(t, x)$ of problem (3.1), (3.2), (4.8). Let

$$
\mathfrak{L}^{(n-1)} u(t, x)=w(t, x),
$$

where $\mathfrak{L}^{(n-1)}$ is a differential operator of the form (3.2). Then

$$
w(0, x)=\mathfrak{L}^{(n-1)} u(0, x)=\sum_{j=0}^{n-1} B^{(j)} \varphi^{(j)}(x),
$$


where the $B^{(j)}$ are differential operators of order $\leq n-1-j$. Since, by condition $(4.8), \varphi^{(j)} \equiv 0$ for all $j=0, \ldots, n-1$, it follows that condition (5.6) is homogeneous.

Therefore, for the function $w$, we obtain the Cauchy problem

$$
\left(\partial_{t}-a^{(n)} \partial_{x}+b^{(n)}\right) w(t, x)=0, \quad w(0, x)=0 .
$$

This problem has the unique solution $w(t, x) \equiv 0$. Consequently, Eq. (5.5) is homogeneous with the operator $\mathfrak{L}^{(n-1)}$ of order $n-1$ and with the homogeneous Cauchy conditions (4.8) for the function $u$. By the inductive assumption, problem (5.5), (4.8) has the unique solution $u(t, x) \equiv 0$.

We have thereby proved the following assertion.

Theorem 5.2. Under the assumptions of Theorem 5.1, there exists a unique classical solution $u \in C^{m}(\bar{Q})$ of the Cauchy problem (2.1), (3.2), (2.2).

\section{ACKNOWLEDGMENTS}

The research was supported by the International Project (0113RK01031) of the Ministry of Education and Science of the Republic Kazakhstan.

\section{REFERENCES}

1. Korzyuk, V.I. and Kozlovskaya, I.S., Solution of the Cauchy Problem for a Hyperbolic Equation with Constant Coefficients in the Case of Two Independent Variables, Differ. Uravn., 2012, vol. 48, no. 5, pp. $700-709$.

2. Korzyuk, V.I. and Kozlovskaya, I.S., Solution of the Cauchy Problem for a Hyperbolic Equation for a Homogeneous Differential Operator in the Case of Two Independent Variables, Dokl. NAN Belarusi, 2011, vol. 55, no. 5, pp. 9-13.

3. Petrovskii, I.G., On the Cauchy Problem for Systems of Linear Partial Differential Equations in a Domain of Nonanalytic Functions, Bull. Moskov. Univ. Mat. Mekh., 1938, vol. 1, no. 7, pp. 1-72.

4. Petrovskii, I.G., Über das Cauchysche Problem für System von partiellen Differentialgleichungen, Mat. Sb., 1937, vol. 2 (44), pp. 815-870.

5. Korzyuk, V.I. and Kozlovskaya, I.S., Cauchy Problem for a Hyperbolic Equation with Constant Coefficients in the Case of Two Independent Variables, in Matematicheskoe modelirovanie $i$ differentsial'nye uravneniya: Tr. Tret'ei mezhdunar. nauchn. konf., 17-22 sentyabrya 2012 g. (Mathematical Modeling and Differential Equations: Proc. 3rd Int. Sci. Conf., September 17-22, 2012), Brest-Minsk, 2012, pp. $171-176$.

6. Korzyuk, V.I., Kozlouskaya, I.S., and Kozlov, A.I., Caushy Problem in Half-Plane for Hyperbolic Equation with Constant Coefficients, in Analytic Methods of Analysis and Differential Equations (AMADE 2012), Cambridge, 2013, pp. 45-71. 